\title{
Exponential Energy Decay for the Kadomtsev-Petviashvili (KP-II) equation
}

\section{Diogo A. Gomes}

Department of Mathematics, CAMGSD, IST 1049-001

Av. Rovisco Pais, Lisbon, Portugal

E-mail address: dgomes@math.ist.utl.pt

\section{Mahendra Panthee}

Center of Mathematics, University of Minho Campus de Gualtar, 4710-057, Braga, Portugal

E-mail address: mpanthee@math.uminho.pt

\begin{abstract}
In this paper we study the exponential decay of the energy of the externally damped Kadomtsev-Petviashvili (KP-II) equation. Our main tool is the classical dissipation-observability method. We use multiplier techniques to establish the main estimates, and obtain exponential decay result.
\end{abstract}

\section{Contents}

1. Introduction

2. Dissipation - observability method

3. Nonlinear KP-II - exponential energy dissipation $\quad 139$

References

2000 Mathematics Subject Classification. 35Q35, 35Q53.

Key words: Dispersive equations, KP equation, Energy decay.

D. Gomes was partially supported by CAMGSD/IST through FCT Program POCTI - FEDER and by grants DENO/FCT-PT/PTDC/EEAACR/67020/2006, PTDC/MAT/69635/2006, PTDC/MAT/72840/2006 and FCTPT/UTAustin/MAT/0057/2008.

M. Panthee was partially supported by the FCT Portugal, through Ciência 2007 program and by the Research Center of Mathematics of the University of Minho, Portugal through the FCT Pluriannual Funding Program. 


\section{Introduction}

Consider the initial value problem (IVP) associated with the KadomtsevPetviashvili (KP) equation,

$$
\left\{\begin{array}{l}
\left(u_{t}+u_{x x x}+u u_{x}\right)_{x}=\alpha u_{y y}, \quad(x, y) \in(0, L) \times(0, L), t \in \mathbb{R} \\
u(x, y, 0)=u_{0}(x, y)
\end{array}\right.
$$

where $u=u(x, y, t)$ is a real valued function, $L>0$ and $\alpha= \pm 1$. This model was derived by Kadomtsev and Petviashvili [8] to describe the propagation of weakly nonlinear long waves on the surface of a fluid, when the wave motion is essentially one-directional with weak transverse effects along y-axis. Equation (1.1) is known as KP-I or KP-II equation depending whether $\alpha=1$ or $\alpha=-1$. In this paper we consider the KP-II equation, that is (1.1) with $\alpha=-1$.

The KP-II equation is a two dimensional generalization of the Kortewegde Vries (KdV) equation

$$
u_{t}+u_{x x x}+u u_{x}=0, \quad x, t \in \mathbb{R},
$$

which arises in modeling the evolution of one dimensional surface gravity waves with small amplitude in a shallow channel of water. The KdV model is a widely studied model which arises in various physical contexts and has a very rich mathematical structure.

It is customary to work with (1.1) for $(x, y) \in \mathbb{R}^{2}$, without any boundary conditions. In this case the $L^{2}$ norm, the energy,

$$
E(u(t)):=\int_{\mathbb{R}^{2}}|u|^{2} d x d y
$$

is a conserved quantity. However, since $(x, y) \in(0, L) \times(0, L)$, if we impose suitable boundary conditions energy may be dissipated. These conditions will be discussed in Section 3. If in addition to these boundary conditions a weak damping is imposed we obtain an exponential decay of the energy. As a damping term, we take a non-negative function $a(x, y)$ and consider the following damped KP-II equation:

$$
\left\{\begin{array}{l}
\left(u_{t}+u_{x x x}+u u_{x}+a u\right)_{x}=\alpha u_{y y}, \quad(x, y) \in(0, L) \times(0, L), t \in \mathbb{R} \\
u(x, y, 0)=u_{0}(x, y) .
\end{array}\right.
$$

If $a>0$ everywhere, an easy computation shows exponential decay. If, however, we allow $a$ to vanish the exponential decay requires additional work. In the case of the KdV equation this problem was studied in [13]. In this paper we extend these results to the KP-II equation by introducing suitable boundary conditions and using the unique continuation principle proved in [12]. We note that our techniques do not rely on the Holmgren's 
Uniqueness Theorem but in a unique continuation principle. Therefore it works both for linear and non-linear equations.

KP models are extensively investigated in the recent literature see for example [2], [4], [5], [6] [7], [9], [11], [12], [14], [15], [16] and references therein, for issues such as local and global well-posedness, gain of regularity and unique continuation principles. As we are interested in the case when the initial data has sufficiently high Sobolev regularity, the question of wellposedness follows, for instance, by the semi-group theory. So, we will omit the details of this aspect.

The plan of the paper is as follows: firstly, in Section 2 we give a compact presentation of the dissipation-observability method which is the basis for many decay results. Then in Section 3 we establish, using multiplier techniques, the main technical estimates which allow us to address the exponential dissipation of energy for the KP-II equation.

\section{Dissipation - observability method}

In this section we put forward a general method to prove energy decay that follows from the energy dissipation law and an observability inequality.

Theorem 2.1. Let $\Omega \subset \mathbb{R}^{n}$ be a domain. Let $A$ be a linear operator, and $B$ a non-linear operator with domain dense in $L^{2}(\Omega)$. Suppose that $u$ is a solution to the evolution equation in $L^{2}(\Omega)$

$$
u_{t}=A u+B(u),
$$

under suitable boundary conditions. Suppose that the evolution associated to (2.1) satisfies a semigroup property and that the energy $E(u(t)):=\int_{\Omega} u^{2} d x$ is dissipated according to

$$
\frac{d}{d t} E(u(t))=-Q(u) \leq 0,
$$

where $\int_{\Omega} u(A u+B(u))=-Q(u)$. Assume that $\forall T>0$ there exists $C>0$ such that the following observability inequality

$$
E(u(\cdot, 0)) \leq C \int_{0}^{T} Q(u(\cdot, s)) d s
$$

holds. Then the energy $E$ decays exponentially, i.e, there exists $\alpha>0$ such that $\forall t \geq 0$,

$$
E(u(t)) \leq C E(u(0)) e^{-\alpha t} .
$$

Proof. Integrating $(2.2)$ in $(0, T)$ we get,

$$
E(u(T))=E(u(0))-\int_{0}^{T} Q(u(s)) d s .
$$


Now, multiplying (2.5) by $C$, adding to itself and using the observability inequality (2.3), yields

$$
\begin{aligned}
(1+C) E(u(T)) & =E(u(0))-C \int_{0}^{T} Q(u(s)) d s+C E(u(0)) \\
& -\int_{0}^{T} Q(u(s)) d s \\
& \leq C E(u(0))-\int_{0}^{T} Q(u(s)) d s \\
& \leq C E(u(0)) .
\end{aligned}
$$

From (2.6) we obtain

$$
E(u(T)) \leq \frac{C}{1+C} E(u(0)) .
$$

Therefore, for some $0<\alpha<1$ we have $E(u(T)) \leq \alpha E(u(0))$. Hence, the semigroup property implies the conclusion of the Theorem.

Lemma 2.2. Let $u$ be as in Theorem 2.1. Then the following estimate holds

$$
E\left(u_{0}\right) \leq \frac{1}{T} \int_{0}^{T} E(u(t))+2 \int_{0}^{T} Q(u) .
$$

Proof. Multiplying (2.1) by $(T-t) u$ and integrating we obtain

$$
\int_{0}^{T} \int_{\Omega}(T-t) u u_{t} d x d t=\int_{0}^{T} \int_{\Omega}(T-t) u(A u+B(u)) d x d t .
$$

After integrating by parts in the $t$ variable, (2.9) yields

i.e.,

$$
\frac{1}{2} \int_{\Omega}\left[\left(\int_{0}^{T} u^{2}\right)-T u_{0}^{2}\right]=-\int_{0}^{T}(T-t) Q(u),
$$

$$
-\frac{T}{2} E\left(u_{0}\right)+\frac{1}{2} \int_{0}^{T} E(u) d t+\int_{0}^{T}(T-t) Q(u) d t=0 .
$$

Now the desired estimate (2.8) follows from (2.10).

Corollary 2.3. Suppose that

$$
\int_{0}^{T} E(u(t)) \leq C(T) \int_{0}^{T} Q(u) .
$$

Then the observability inequality (2.3) holds. 


\section{Nonlinear KP-II - exponential energy dissipation}

Let $a(x, y) \geq a_{0}>0$ almost everywhere in the complement of a compact non-empty proper subset $\Theta$ of $\Omega:=(0, L) \times(0, L)$. We assume that for some $\delta>0, \Theta \subset(\delta, L-\delta) \times(\delta, L-\delta)$, so that we can apply the UCP, using an extension technique.

Consider the damped KP-II model

$$
\left\{\begin{array}{l}
u_{t}+u_{x x x}+\partial_{x}^{-1} u_{y y}+u u_{x}+a(x, y) u=0,(x, y) \in(0, L) \times(0, L), t \in \mathbb{R}, \\
u(L, y, t)=0=u(0, y, t), \quad u(x, L, t)=0=u(x, 0, t), \\
u_{x}(L, y, t)=0, \\
u(x, y, 0)=u_{0}(x, y),
\end{array}\right.
$$

where the operator $\partial_{x}^{-1}$ is defined as $\partial_{x}^{-1} f(x, y, t)=g(x, y, t)$ with $g(L, y, t)=0$ and $g_{x}(x, y, t)=f(x, y, t)$.

Define the energy as

$$
E(u(t)):=\frac{1}{2} \int_{0}^{L} \int_{0}^{L} u^{2}(x, y, t) d x d y .
$$

As we prove in the next proposition, the energy is a decreasing function of $t$. Our main objective is to show that the decay is exponential in time, by using the dissipation-observability method.

Proposition 3.1. Suppose $u$ solves (3.1), and let $E$ be given by (3.2). Then

$$
\begin{aligned}
\frac{d}{d t} E(u(t)) & =-\frac{1}{2} \int_{0}^{L}\left[u_{x}^{2}(0, y, t)+\left(\partial_{x}^{-1} u_{y}\right)^{2}(0, y, t)\right] d y \\
& -\int_{0}^{L} \int_{0}^{L} a(x, y) u^{2}(x, y) d x d y \leq 0 .
\end{aligned}
$$

Proof. We have

$$
\begin{aligned}
\frac{d}{d t} E(u(t)) & =\frac{1}{2} \frac{d}{d t} \int_{0}^{L} \int_{0}^{L} u^{2}(x, y, t) d x d y \\
& =\int_{0}^{L} \int_{0}^{L} u u_{t}(x, y, t) d x d y \\
& =\int_{0}^{L} \int_{0}^{L} u\left(-u_{x x x}-\partial_{x}^{-1} u_{y y}-u u_{x}-a(x, y) u\right) d x d y .
\end{aligned}
$$


Now observe that

$$
\begin{aligned}
-\int_{0}^{L} \int_{0}^{L} u u_{x x x} d x d y & =\int_{0}^{L}\left[\int_{0}^{L} u_{x} u_{x x} d x-\left.u u_{x x}\right|_{x=0} ^{L}\right] d y \\
& =\int_{0}^{L}\left[\frac{1}{2} \int_{0}^{L}\left(u_{x}\right)_{x}^{2} d x\right] d y \\
& =\frac{1}{2} \int_{0}^{L}\left[\left.u_{x}^{2}\right|_{x=0} ^{L}\right] d y \\
& =-\frac{1}{2} \int_{0}^{L} u_{x}^{2}(0, y, t) d y .
\end{aligned}
$$

Define $v$ by $u_{y}=v_{x}$, then

$$
\begin{aligned}
-\int_{0}^{L} \int_{0}^{L} u \partial_{x}^{-1} u_{y y} d x d y & =-\int_{0}^{L} \int_{0}^{L} u v_{y} d y d x \\
& =\int_{0}^{L}\left[\int_{0}^{L} u_{y} v d y-\left.u v\right|_{x=0} ^{L}\right] d x \\
& =\int_{0}^{L} \int_{0}^{L} v_{x} v d x d y \\
& =\frac{1}{2} \int_{0}^{L} \int_{0}^{L}\left(v^{2}\right)_{x} d x d y \\
& =\frac{1}{2} \int_{0}^{L}\left[v^{2}(L, y, t)-v^{2}(0, y, t)\right] d y \\
& =-\frac{1}{2} \int_{0}^{L} v^{2}(0, y, t) d y
\end{aligned}
$$

where we have used $v=0$ at $(L, y, t)$.

Also, integrating by parts yields

$$
-\int_{0}^{L} \int_{0}^{L} u^{2} u_{x} d x d y=0
$$

Now, using (3.5), (3.6) and (3.7) in (3.4) we obtain (3.3).

Now we state and prove the main result of this work that deals with the exponential decay of energy of the nonlinear KP-II equation.

Theorem 3.2. Given $M>0$, let $u$ be a solution of (3.1) with data $u_{0} \in$ $H^{s}\left(\mathbb{R}^{2}\right), s \geq 3$, satisfying $\left\|u_{0}\right\|_{L^{2}(\Omega)} \leq M$, and let $E(u(t))$ be the energy as defined in (3.2). Then the energy $E(u(t))$ decays exponentially. 
Proof. The proof of this Theorem follows from Theorem 2.1, using Corollary 2.3 with

$Q(u)=\frac{1}{2} \int_{0}^{L}\left[u_{x}^{2}(0, y, t)+\left(\partial_{x}^{-1} u_{y}\right)^{2}(0, y, t)\right] d y+\int_{0}^{L} \int_{0}^{L} a(x, y) u^{2}(x, y) d x d y$

and the estimate

$$
\begin{aligned}
\int_{0}^{T} \int_{0}^{L} \int_{0}^{L}|u|^{2} \leq \tilde{C} & \left\{\int_{0}^{T} \int_{0}^{L}\left[\left|u_{x}(0, y, t)\right|^{2}+\left|\partial_{x}^{-1} u_{y}(0, y, t)\right|^{2}\right] d y d t\right. \\
& \left.+2 \int_{0}^{T} \int_{0}^{L} \int_{0}^{L} a(x, y)|u|^{2} d x d y d t\right\}
\end{aligned}
$$

which holds for some $\tilde{C}>0$ independent of solution $u$ to (3.1) with initial data $u_{0}$ satisfying $\left\|u_{0}\right\|_{L^{2}(\Omega)} \leq M$ for any given $M>0$, as will be shown in Lemma 3.5 below. (3.8).

Now we prove the following result which will be used in the proof of

Lemma 3.3. Let $u$ be a solution of (3.1). Then the following estimate holds:

$$
\|u\|_{L^{2}\left(0, T ; H^{1}(\Omega)\right)}^{2} \leq \frac{2 L}{3}\left\|u_{0}\right\|_{L^{2}(\Omega)}^{2}+\frac{4 C T}{81}\left\|u_{0}\right\|_{L^{2}(\Omega)}^{3} .
$$

Proof. Multiply equation (3.1) by $x u$ and integrate on $(0, L) \times(0, L) \times(0, T)$. The resulting identity is composed of five terms, that we simplify next:

$$
\begin{aligned}
\int_{0}^{T} \int_{0}^{L} \int_{0}^{L} x_{0} d x d y d t= & \frac{1}{2} \int_{0}^{T} \int_{0}^{L} \int_{0}^{L} x \frac{d}{d t}\left(u^{2}\right) d t d x d y \\
= & \frac{1}{2} \int_{0}^{L} \int_{0}^{L} x u^{2}(x, y, T) d x d y \\
& -\frac{1}{2} \int_{0}^{L} \int_{0}^{L} x u_{0}^{2}(x, y) d x d y
\end{aligned}
$$


the next term is

$$
\begin{aligned}
\int_{0}^{T} \int_{0}^{L} \int_{0}^{L} x u u_{x x x}= & \int_{0}^{T} \int_{0}^{L}\left[-\int_{0}^{L}(x u)_{x} u_{x x} d x+\left.x u u_{x x}\right|_{0} ^{L}\right] d y d t \\
= & \int_{0}^{T} \int_{0}^{L}\left[-\int_{0}^{L} u u_{x x} d x-\int_{0}^{L} x u_{x} u_{x x} d x\right] d y d t \\
= & \int_{0}^{T} \int_{0}^{L}\left[\int_{0}^{L} u_{x}^{2} d x-\left.u u_{x}\right|_{0} ^{L}\right. \\
& \left.+\int_{0}^{L}\left(x u_{x}\right)_{x} u_{x} d x-\left.x u_{x}^{2}\right|_{0} ^{L}\right] d y d t \\
= & \int_{0}^{T} \int_{0}^{L}\left[\int_{0}^{L} u_{x}^{2} d x+\int_{0}^{L} u_{x}^{2} d x+\int_{0}^{L} x u_{x x} u_{x} d x\right] d y d t \\
= & \int_{0}^{T} \int_{0}^{L}\left[2 \int_{0}^{L} u_{x}^{2} d x-\int_{0}^{L}\left(x u_{x x}\right)_{x} u d x+\left.x u_{x x} u\right|_{0} ^{L}\right] d y d t \\
= & \int_{0}^{T} \int_{0}^{L}\left[2 \int_{0}^{L} u_{x}^{2} d x-\int_{0}^{L} u_{x x} u d x-\int_{0}^{L} x u_{x x x} u d x\right] d y d t \\
= & \int_{0}^{T} \int_{0}^{L}\left[2 \int_{0}^{L} u_{x}^{2} d x+\int_{0}^{L} u_{x}^{2} d x-\left.u_{x} u\right|_{0} ^{L}\right. \\
& \left.-\int_{0}^{L} x u_{x x x} u d x\right] d y d t
\end{aligned}
$$

which, from (3.11), yields

$$
\int_{0}^{T} \int_{0}^{L} \int_{0}^{L} x u u_{x x x} d x d y d t=\frac{3}{2} \int_{0}^{T} \int_{0}^{L} \int_{0}^{L} u_{x}^{2} d x d y d t
$$

for the next term, as earlier, we set $u_{y}=v_{x}$, and obtain

$$
\int_{0}^{T} \int_{0}^{L} \int_{0}^{L} x u \partial_{x}^{-1} u_{y y} d x d y d t=\frac{1}{2} \int_{0}^{T} \int_{0}^{L} \int_{0}^{L} v^{2} d x d y d t .
$$

Also, integrating by parts we get,

$$
\begin{aligned}
\int_{0}^{T} \int_{0}^{L} \int_{0}^{L} \text { xuuu }_{x} d x d y d t & =\frac{1}{3} \int_{0}^{T} \int_{0}^{L} \int_{0}^{L} x\left(u^{3}\right)_{x} d x d y d t \\
& =-\frac{1}{3} \int_{0}^{T} \int_{0}^{L} \int_{0}^{L} u^{3} d x d y d t .
\end{aligned}
$$

Finally, the last term is simply

$$
\int_{0}^{T} \int_{0}^{L} \int_{0}^{L} x a(x, y) u^{2} d x d y d t .
$$

São Paulo J.Math.Sci. 5, 2 (2011), 135-148 
Now, adding (3.10), (3.12),(3.13), (3.14) and (3.15) we get

$$
\begin{gathered}
\frac{1}{2} \int_{0}^{L} \int_{0}^{L} x u^{2}(x, y, T) d x d y-\frac{1}{2} \int_{0}^{L} \int_{0}^{L} x u_{0}^{2}(x, y) d x d y+\frac{3}{2} \int_{0}^{T} \int_{0}^{L} \int_{0}^{L} u_{x}^{2} d x d y d t \\
+\int_{0}^{T} \int_{0}^{L} \int_{0}^{L} x a(x, y) u^{2} d x d y d t+\frac{1}{2} \int_{0}^{T} \int_{0}^{L} \int_{0}^{L} v^{2} d x d y d t \\
-\frac{1}{3} \int_{0}^{T} \int_{0}^{L} \int_{0}^{L} u^{3} d x d y d t=0 .
\end{gathered}
$$

Or,

$$
\begin{aligned}
\frac{3}{2} \int_{0}^{T}\|u\|_{H^{1}(\Omega)}^{2} d t= & \frac{1}{2} \int_{0}^{L} \int_{0}^{L} x u_{0}^{2}(x, y) d x d y-\frac{1}{2} \int_{0}^{L} \int_{0}^{L} x u^{2}(x, y, T) d x d y \\
& -\int_{0}^{T} \int_{0}^{L} \int_{0}^{L} x a(x, y) u^{2} d x d y d t-\frac{1}{2} \int_{0}^{T} \int_{0}^{L} \int_{0}^{L} v^{2} d x d y d t \\
& +\frac{1}{3} \int_{0}^{T} \int_{0}^{L} \int_{0}^{L} u^{3} d x d y d t,
\end{aligned}
$$

which yields,

$$
\begin{aligned}
\int_{0}^{T}\|u\|_{H^{1}(\Omega)}^{2} d t= & \frac{1}{3} \int_{0}^{L} \int_{0}^{L} x u_{0}^{2}(x, y) d x d y-\frac{1}{3} \int_{0}^{L} \int_{0}^{L} x u^{2}(x, y, T) d x d y \\
& -\frac{2}{3} \int_{0}^{T} \int_{0}^{L} \int_{0}^{L} x a(x, y) u^{2} d x d y d t-\frac{1}{3} \int_{0}^{T} \int_{0}^{L} \int_{0}^{L} v^{2} d x d y d t \\
& +\frac{2}{9} \int_{0}^{T} \int_{0}^{L} \int_{0}^{L} u^{3} d x d y d t .
\end{aligned}
$$

Since $a(x, y) \geq 0$, we obtain from (3.18) that

$$
\int_{0}^{T}\|u\|_{H^{1}(\Omega)}^{2} d t \leq \frac{L}{3}\left\|u_{0}\right\|_{L^{2}(\Omega)}^{2}+\frac{2}{9} \int_{0}^{T} \int_{0}^{L} \int_{0}^{L} u^{3} d x d y d t .
$$

We have the following Gagliardo-Nirenberg type inequality (see [3] or [10])

$$
\|u\|_{L^{3}(\Omega)} \leq C\|u\|_{\dot{H}^{1}(\Omega)}^{\frac{1}{3}}\|u\|_{L^{2}(\Omega)}^{\frac{2}{3}} \leq C\|u\|_{H^{1}(\Omega)}^{\frac{1}{3}}\|u\|_{L^{2}(\Omega)}^{\frac{2}{3}} .
$$


Now using (3.20) and (3.3) the last term in (3.19) can be controlled by

$$
\begin{aligned}
\int_{0}^{T} \int_{0}^{L} \int_{0}^{L} u^{3} d x d y d t & \leq \int_{0}^{T}\|u\|_{H^{1}(\Omega)}\|u\|_{L^{2}(\Omega)}^{2} d t \\
& \leq C\left\|u_{0}\right\|_{L^{2}(\Omega)}^{2} \int_{0}^{T}\|u\|_{H^{1}(\Omega)} d t \\
& \leq C\left\|u_{0}\right\|_{L^{2}(\Omega)}^{2} \sqrt{T}\|u\|_{L^{2}\left(0, T ; H^{1}(\Omega)\right)}
\end{aligned}
$$

Substituting (3.21) in (3.19) yields

$$
\begin{aligned}
\|u\|_{L^{2}\left(0, T ; H^{1}(\Omega)\right)}^{2} & \leq \frac{L}{3}\left\|u_{0}\right\|_{L^{2}(\Omega)}^{2}+C \frac{2}{9} \sqrt{T}\left\|u_{0}\right\|_{L^{2}(\Omega)}^{2}\|u\|_{L^{2}\left(0, T ; H^{1}(\Omega)\right)} \\
& \leq \frac{L}{3}\left\|u_{0}\right\|_{L^{2}(\Omega)}^{2}+\frac{\left(\frac{2}{9} C \sqrt{T}\left\|u_{0}\right\|_{L^{2}(\Omega)}^{2}\right)^{2}}{2}+\frac{\left(\|u\|_{L^{2}\left(0, T ; H^{1}(\Omega)\right)}\right)^{2}}{2} \\
& \leq \frac{L}{3}\left\|u_{0}\right\|_{L^{2}(\Omega)}^{2}+\frac{2 C T}{81}\left\|u_{0}\right\|_{L^{2}(\Omega)}^{4}+\frac{1}{2}\|u\|_{L^{2}\left(0, T ; H^{1}(\Omega)\right)}^{2} .
\end{aligned}
$$

Therefore, from (3.22) we get

$$
\|u\|_{L^{2}\left(0, T ; H^{1}(\Omega)\right)}^{2} \leq \frac{2 L}{3}\left\|u_{0}\right\|_{L^{2}(\Omega)}^{2}+\frac{4 C T}{81}\left\|u_{0}\right\|_{L^{2}(\Omega)}^{4},
$$

which yields the required result.

In addition to the Lemma 3.3, unique continuation principle (UCP) for the KP-II equation (1.1) will also be used in the proof the estimate (3.8). The UCP for the KP-II equation (1.1) was established in [12] whose precise statement is given in the following theorem.

Theorem $3.4([12])$. Let $u \in C\left(\mathbb{R} ; H^{s}\left(\mathbb{R}^{2}\right)\right)$ be a solution to the IVP associated with the KP-II equation with $s>0$ large enough. If there exists a non trivial time interval $I=[-T, T]$ such that for some $\beta>0$

$$
\operatorname{supp} u(t) \subseteq[-\beta, \beta] \times[-\beta, \beta], \quad \forall t \in I,
$$

then $u \equiv 0$.

In the following lemma we prove the main estimate (3.8) used in the proof of Theorem 3.2. 
Lemma 3.5. given $M>0$, there exists a constant $\tilde{C}>0$ such that the following estimate holds

$$
\begin{array}{r}
\int_{0}^{T} \int_{0}^{L} \int_{0}^{L}|u|^{2} \leq \tilde{C}\left\{\int_{0}^{T} \int_{0}^{L}\left[\left|u_{x}(0, y, t)\right|^{2}+\left|\partial_{x}^{-1} u_{y}(0, y, t)\right|^{2}\right] d y d t\right. \\
\left.+2 \int_{0}^{T} \int_{0}^{L} \int_{0}^{L} a(x, y)|u|^{2} d x d y d t\right\}
\end{array}
$$

for all solutions $u$ of (3.1) with initial data $u_{0}$ satisfying $\left\|u_{0}\right\|_{L^{2}(\Omega)} \leq M$.

Proof. We prove it by contradiction with use of the Lemma 3.3 and the unique continuation principle stated in Theorem 3.4.

Suppose that (3.24) is false. Then there exists a sequence of solution $u_{n}$ of (3.1) such that

$$
\lim _{n \rightarrow \infty} \frac{\left\|u_{n}\right\|_{L^{2}\left(0, T ; L^{2}(\Omega)\right)}^{2}}{B_{n}}=+\infty
$$

where

$$
\begin{aligned}
B_{n}= & \int_{0}^{T} \int_{0}^{L}\left[\left|\partial_{x} u_{n}(0, y, t)\right|^{2}+\left|\partial_{x}^{-1} \partial_{y} u_{n}(0, y, t)\right|^{2}\right] d y d t \\
& +2 \int_{0}^{T} \int_{0}^{L} \int_{0}^{L} a(x, y)\left|u_{n}\right|^{2} d x d y d t .
\end{aligned}
$$

Let

$$
\lambda_{n}=\left\|u_{n}\right\|_{L^{2}\left(0, T ; L^{2}(\Omega)\right)} \quad \text { and } \quad v_{n}(x, y, t)=\frac{1}{\lambda_{n}} u_{n}(x, y, t) .
$$

From Lemma 3.3, we have that $\lambda_{n}$ is a bounded sequence for $\left\|u_{n}(0)\right\|_{L^{2}(\Omega)} \leq$ $M$. Therefore, extracting a subsequence if necessary, we can assume that $\lambda_{n} \rightarrow \lambda \geq 0$.

We notice that $v_{n}$ solves

$$
\left(v_{n}\right)_{t}+\left(v_{n}\right)_{x x x}+\partial_{x}^{-1}\left(v_{n}\right)_{y y}+\lambda_{n}\left(v_{n}\right)\left(v_{n}\right)_{x}+a(x, y)\left(v_{n}\right)=0,
$$

for $(x, y) \in \Omega, t \in \mathbb{R}$, together with boundary conditions

$$
\left\{\begin{array}{l}
\left(v_{n}\right)(L, y, t)=0=\left(v_{n}\right)(0, y, t), \quad\left(v_{n}\right)(x, L, t)=0=\left(v_{n}\right)(x, 0, t), \\
\left(v_{n}\right)_{x}(L, y, t)=0,
\end{array}\right.
$$

with initial data $\frac{1}{\lambda_{n}} u_{n}(x, y, 0)$. Moreover,

$$
\left\|v_{n}\right\|_{L^{2}\left(0, T ; L^{2}(\Omega)\right)}=1
$$


and from (3.25)

$$
\begin{array}{r}
\int_{0}^{T} \int_{0}^{L}\left[\left|\partial_{x} v_{n}(0, y, t)\right|^{2}+\left|\partial_{x}^{-1} \partial_{y} u_{n}(0, y, t)\right|^{2}\right] d y d t \\
+2 \int_{0}^{T} \int_{0}^{L} \int_{0}^{L} a(x, y)\left|v_{n}\right|^{2} d x d y d t \rightarrow 0,
\end{array}
$$

as $n \rightarrow \infty$.

In view of Lemma $2.2, v_{n}(x, y, 0)$ is bounded in $L^{2}(\Omega)$. Thus, combining with an analogue of (2.8), we have

$$
\left\|v_{n}(\cdot, \cdot, t)\right\|_{L^{2}(\Omega)} \leq M, \quad \forall 0 \leq t \leq T .
$$

Now, from Lemma 3.3,

$$
\left\|v_{n}\right\|_{L^{2}\left(0, T ; H^{1}(\Omega)\right)}^{2} \leq C,
$$

for all $n \in \mathbb{N}$.

Estimates (3.28) and (3.31) yield

$$
\left(v_{n}\right)_{t}=-\left(v_{n}\right)_{x x x}-\partial_{x}^{-1}\left(v_{n}\right)_{y y}-\lambda_{n} v_{n}\left(v_{n}\right)_{x}-a(x, y) v_{n}
$$

is bounded in $L^{2}\left(0, T ; H^{-2}(\Omega)\right)$. Note that the non-linear term, is bounded in $L^{2}\left(0, T ; L^{p}(\Omega)\right)$ for all $1 \leq p<2$, which is bounded in $L^{2}\left(0, T ; H^{-2}(\Omega)\right)$.

Since the embedding $H^{1}(\Omega) \hookrightarrow L^{2}(\Omega)$ is compact, by Rellich's theorem, it follows that $\left\{v_{n}\right\}$ is relatively compact in $L^{2}\left(0, T ; L^{2}(\Omega)\right)$. By extracting a subsequence we may deduce that

$$
v_{n j} \rightarrow v, \quad \text { weakly in } \quad L^{2}\left(0, T ; H^{1}(\Omega)\right) \cap H^{1}\left(0, T ; H^{-2}(\Omega)\right)
$$

and

$$
v_{n j} \rightarrow v, \quad \text { strongly in } \quad L^{2}\left(0, T ; L^{2}(\Omega)\right) .
$$

Since $\left\|v_{n j}\right\|_{L^{2}\left(0, T ; L^{2}(\Omega)\right)}=1$, it follows that

$$
\|v\|_{L^{2}\left(0, T ; L^{2}(\Omega)\right)}=1 .
$$


By weak lower semicontinuity of convex functionals we have (see (3.29))

$$
\begin{gathered}
0=\liminf _{j \rightarrow \infty}\left\{\int_{0}^{T} \int_{0}^{L}\left[\left|\partial_{x} v_{n j}(0, y, t)\right|^{2}+\left|\partial_{x}^{-1} \partial_{y} v_{n j}(0, y, t)\right|^{2}\right] d y d t\right. \\
\left.+2 \int_{0}^{T} \int_{0}^{L} \int_{0}^{L} a(x, y)\left|v_{n j}\right|^{2} d x d y d t\right\} \\
\geq \int_{0}^{T} \int_{0}^{L}\left[\left|\partial_{x} v(0, y, t)\right|^{2}+\left|\partial_{x}^{-1} \partial_{y} v(0, y, t)\right|^{2}\right] d y d t \\
+2 \int_{0}^{T} \int_{0}^{L} \int_{0}^{L} a(x, y)|v|^{2} d x d y d t
\end{gathered}
$$

From this we conclude that $a(x, y) v \equiv 0$ in $\Omega \times(0, T)$. Since $a(x, y)>0$ in $\Theta^{c}$, so in particular $v \equiv 0$ in $\Theta^{c} \times(0, T)$. We will show that $v \equiv 0$ in $\Omega \times(0, T)$.

Note that the limit $v$ satisfies

$$
v_{t}+v_{x x x}+\partial_{x}^{-1} v_{y y}+\lambda v v_{x}=0
$$

where $\lambda \geq 0$ is the limit of $\lambda_{n}$ as $n \rightarrow \infty$.

In any case, whether $\lambda=0$ or $\lambda>0$, we will use the unique continuation property discussed earlier to conclude that $v \equiv 0$ in $\Omega \times(0, T)$. To be able to apply the unique continuation property, we must find an smooth extension of $v$ in $\mathbb{R}^{2}$. Let $Z:=(\delta, L-\delta) \times(\delta, L-\delta)$ and define a function,

$$
w(x, y, t)= \begin{cases}v(x, y, t), & (x, y, t) \in Z \times(0, T), \\ 0, & (x, y, t) \in\left\{\mathbb{R}^{2}-Z\right\} \times(0, T) .\end{cases}
$$

Because $\Theta \subset(\delta, L-\delta) \times(\delta, L-\delta)$, this extension is as smooth as $v$. Furthermore $w$ satisfies

$$
\left\{\begin{array}{l}
w_{t}+w_{x x x}+\partial_{x}^{-1} w_{y y}+\lambda w w_{x}=0, \quad(x, y, t) \in \mathbb{R}^{2} \times(0, T) \\
w(x, y, 0)=\phi(x, y)
\end{array}\right.
$$

where

$$
\phi(x, y)= \begin{cases}v(x, y, 0), & (x, y) \in Z \\ 0, & (x, y) \in \mathbb{R}^{2}-Z .\end{cases}
$$

Note that, $\phi$ is a compactly supported function in $H^{s}\left(\mathbb{R}^{2}\right), s \geq 3$. So, by the regularization property of the KP-II equation (see [9]), the IVP (3.39) has a smooth solution $w$. Therefore, by the unique continuation property (Theorem 3.4), we conclude that $w \equiv 0$ in $\Omega \times(0, T)$. Consequently we conclude that $v \equiv 0$ in $\Omega \times(0, T)$ which contradicts (3.35). Hence (3.24) must be true. 


\section{References}

[1] J. Bourgain, On the Compactness of the Support of Solutions of Dispersive Equations, IMRN International Mathematics Research Notices 1997, No. 9 437-447.

[2] J. Bourgain, On the Cauchy problem for the Kadomtsev-Petviashvili equation, GAFA, 3 (1993) 315-341.

[3] A. Friedman, Partial Differential Equations, Holt, Rinehart and Winston, New York, 1976.

[4] R. J. Iório Jr., W. V. L. Nunes, On equations of KP-type, Proc. Roy. Soc., (1998) 725-743.

[5] P. Isaza, J. Meija, Global solution for the Kadomtsev-Petviashvili equation (KP-II) in anisotropic Sobolev spaces of negative indices, Electronic Journal of Differential Equations, 2003 (2003) 1-12.

[6] P. Isaza, J. Meija, Local and Global Cauchy problem for the KadomtsevPetviashvili (KP-II) equation in Sobolev spaces of negative indices, Comm. PDE, 26 (2001) 1027-1057.

[7] P. Isaza, J. Meija, The Cauchy problem for the Kadomtsev-Petviashvili equation (KP-II) in Sobolev spaces $H^{s}, s>0$, Differential and Integral Eqns., 14 (2001) 529-557.

[8] B. B. Kadomtsev, V. I. Petviashvili, On the stability of solitary waves in weakly dispersive media, Sov. Phys. Dokl., 15 (1970) 539-549.

[9] J. L. Levandosky, Gain of regularity for the KP-II equation, Indiana Univ. Math. J., 49 (1) (2000) 353-403.

[10] F. Linares, G. Ponce, Introduction to nonlinear dispersive equations, Universitext, Springer, New York, 2009.

[11] L. Molinet, J. C. Saut, N. Tzvetkov, Global well-posedness for the KP-I equation, Math. Ann. 324 (2002) 255-275.

[12] M. Panthee, Unique continuation property for the Kadomtsev-Petviashvili (KP-II) equation, Electronic Journal of Differential Equations, Vol 2005(2005), No. 59, 1-12.

[13] G. Perla Menzala, C. F. Vasconcellos, E. Zuazua Stabilization of the Korteweg-de Vries equation with localized damping, Quart. Appl. Math. 60 (2002), no. 1, 111-129.

[14] H. Takaoka, Global well-posedness for the Kadomtsev-Petviashvili II equation, Discrete Contin. Dynam. Systems 6 (2000) 483-449.

[15] H. Takaoka, N. Tzvetkov, On the local regularity of KadomtsevPetviashvili-II equation, IMRN, International Mathematics Research Notices 2 (2001) 77-114.

[16] N. Tzvetkov, Global low regularity solutions for Kadomtsev-Petviashvili equation, Differential and Integral Equations 13 (2000) 1289-1320. 